\title{
Susceptibility testing of Mycobacterium tuberculosis to pyrazinamide
}

Pyrazinamide (PZA) is one of four first-line antituberculosis drugs administered during the 2-month intensive phase of initial therapy for newly diagnosed tuberculosis (TB) patients. PZA plays a special role in this standard treatment regimen by affecting the semidormant subpopulation of Mycobacterium tuberculosis persisting in an acidic environment, perhaps in the early inflammation sites [1]. More detailed information about this unique antimicrobial agent active against $M$. tuberculosis can be found in a recent review [2].

Proper management of TB patients requires susceptibility testing of the patients' pretreatment isolates to detect primary drug resistance, and the testing of cultures obtained during the course of therapy to identify acquired drug resistance. Susceptibility testing of $M$. tuberculosis to three of the four first-line drugs isoniazid, rifampin and ethambutol (or streptomycin) does not represent any serious problems in a qualified mycobacteriology laboratory. The situation with PZA is quite different; no reliable procedures for a test with this drug were available until very recently. The difficulties in developing an appropriate procedure were associated with attempts to perform such a test with PZA concentrations attainable in blood or tissue, i.e., $16-60 \mu \mathrm{g} / \mathrm{ml}$. These low concentrations are active in vitro against $M$. tuberculosis only at $\mathrm{pH} 5.5-5.6$ [3-7]. The problem is that such an acidic environment is quite unfavourable for $M$. tuberculosis, and $\geqslant 10 \%$ isolates will not grow at all at this $\mathrm{pH}[8,9]$. Several attempts have been made to overcome the problem $[10,11]$.

A pragmatic solution was suggested in a report that showed that PZA can exhibit the inhibitory effect in $7 \mathrm{H} 12$ broth at $\mathrm{pH}$ 6.0-6.2, under the conditions such that the drug concentration is increased up to 300 or $400 \mu \mathrm{g} / \mathrm{ml}$ [7]. It appeared that $\mathrm{pH} 6.0-6.2$ of $7 \mathrm{H} 12$ broth was within the optimal range for M. tuberculosis cultivation, and some strains grew even better at this $\mathrm{pH}$ than at the conventional $\mathrm{pH} 6.8$ [7]. Application of concentrations much higher than those attainable in vivo excludes any attempts to correlate the test results with the pharmacokinetic parameters. Instead, such a test should have only one goal - distinction between PZA-susceptible and PZA-resistant strains. This approach places PZA into the situation which already has been accepted for other anti-TB drugs, for which the so-called critical concentrations may greatly exceed those attainable in in vivo. An example of this is the critical concentration of rifampin in the egg-based culture media. Based on this approach, the manufacturer of the Bactec-460 system suggested a procedure with $100 \mu \mathrm{g}$ of $\mathrm{PZA} / \mathrm{ml}$ in $7 \mathrm{H} 12$ broth at $\mathrm{pH} 6.0$ [9]. More reliable distinction between PZA-susceptible and PZA-resistant strains was observed when $300 \mu \mathrm{g} / \mathrm{ml}$ of PZA was used as a critical concentration [12], or when three concentrations $(100,300,900 \mu \mathrm{g} / \mathrm{ml})$ were employed to determine the MIC of the drug with corresponding reports as 'susceptible', 'intermediate', or 'resistant' [13]. Another recently developed and less expensive option is a special agar medium with $\mathrm{pH} 6.2$ with a PZA concentration of 900 or $1200 \mu \mathrm{g} / \mathrm{ml}$ [14].

Many clinical laboratories have been testing $M$. tuberculosis isolates with PZA after introduction of the Bactec technique. Along with this, controversies and unresolved questions have emerged [15-17]. The article by Zhang et al. [18] published in this issue of the Journal addresses these issues, particularly the problem of false resistance reports. One of the factors that may affect the results is the inoculum size. Zhang and his colleagues have shown that inocula of $\geqslant 10^{7} \mathrm{cfu} / \mathrm{ml}$ increase the $\mathrm{pH}$ of the agar medium from 5.6 to 6.6 or more, which made the PZA concentration of $60 \mu \mathrm{g} / \mathrm{ml}$ inactive against $M$. tuberculosis. This finding confirms the previous observation [5] that excessive inoculum can lead to alkalisation of the medium, which is known as one of the causes of false resistance. Another alkalising factor, reported by Zhang et al., is bovine serum albumin (BSA) - usually present in the culture medium at a concentration of $0.5 \%$. Fetal bovine serum (FBS), a BSA-containing product, at a concentration of $10 \%$, changed the intended $\mathrm{pH}$ of the medium from 5.5 to 6.5 , with a 10 -fold increase of the MIC (from $50 \mu \mathrm{g} / \mathrm{ml}$ at $\mathrm{pH}$ 5.5). The authors have suggested that the negative impact of BSA is also related to the ability of FBS to bind with pyrazinoic acid (POA), the active moiety of PZA. Either BSA or BSA-containing products are essential components of culture media designed for $M$. tuberculosis cultivation. Therefore, the only solution to the problem is the previously suggested increase of the PZA critical concentration up to levels still active in more alkaline conditions than the $\mathrm{pH} 5.5$ environment. 
Zhang et al. have made theoretical assumptions based on the Henderson-Hasselbach equation in regard to concentrations of PZA needed to inhibit growth of $M$. tuberculosis at different $\mathrm{pH}$ values. The prediction from the Henderson-Hasselbach equation indicated that, at $\mathrm{pH} 6.0$, the MIC of PZA should be $200 \mu \mathrm{g} / \mathrm{ml}$. Based on this calculation and taking into account the abovementioned possibilities of some alkalisation of the medium, the actual $\mathrm{pH}$ of the $7 \mathrm{H} 12$ broth being used for the Bactec-460 system may somehow be higher than the intended $\mathrm{pH}$ 6.0. These facts may explain the reports of false resistance when a concentration of $100 \mu \mathrm{g} / \mathrm{ml}$ was used in this system [15-17]. It was also reported that strains that did not have any $p n c A$ mutations and were resistant to $100 \mu \mathrm{g} / \mathrm{ml}$ at initial testing appeared to be susceptible to PZA by retesting [19]. Information provided by Zhang and his colleagues $[18,19]$ supports the previous suggestions to use PZA at $300 \mu \mathrm{g} / \mathrm{ml}$ instead of $100 \mu \mathrm{g} / \mathrm{ml}$ in a single concentration test by the Bactec method in $\mathrm{pH} 6.0$ medium $[2,7,13]$. For the same reason, PZA concentrations of 900 or $1200 \mu \mathrm{g} / \mathrm{ml}$ have been suggested for the new agar medium with pH 6.2 that contains $10 \%$ of FBS [14].

The paper by Zhang et al. alerts the clinical laboratories to possible causes of discrepancies, especially when attempts are made to perform such a procedure at a very acidic $\mathrm{pH}(5.5-5.6)$, or when the PZA concentration is too low for the chosen $\mathrm{pH}$ conditions. Possible alkalisation of the medium versus initial $\mathrm{pH}$ during cultivation should be taken into account when the PZA critical concentration is designed. It is quite possible that most of the strains without any genetic alterations in their DNA reported in the literature as resistant to $100 \mu \mathrm{g} / \mathrm{ml}$, may not have been resistant to PZA at all. There is now enough evidence to show that a test at $\mathrm{pH}$ 6.0-6.2 with $300 \mu \mathrm{g} / \mathrm{ml}$ (or with 300 and $900 \mu \mathrm{g} / \mathrm{ml}$ ) in $7 \mathrm{H} 12$ broth, as well as with 900 or $1200 \mu \mathrm{g} / \mathrm{ml}$ in the new agar medium, would provide the most reliable reports for distinguishing PZA-susceptible and PZA-resistant M. tuberculosis isolates.

LEONID HEIFETS

Mycobacteriology Reference Clinical Laboratory and Kramer Foundation Professor in Mycobacteriology at National Jewish Medical and Research Center and University of Colorado Health Sciences Center, Denver, CO 80206, USA (e-mail: HeifetsL@njc.org)

\section{References}

1. Mitchison DA. The action of antituberculosis drugs in shortcourse chemotherapy. Tubercle 1985; 66: 219-225.

2. Heifets LB. Antimicrobial agents: pyrazinamide. In: Yu VL, Merigan TC, Barriere S-L (eds) Antimicrobial therapy and vaccines. Baltimore, Williams \& Wilkins (Waverly Company). 1999: 668-676.

3. Dickinson JM, Mitchison DA. Observations in vitro on the suitability of pyrazinamide for intermittent chemotherapy of tuberculosis. Tubercle 1970; 51: 389-396.

4. Konno K, Feldman FM, McDermott W. Pyrazinamide susceptibility and amidase activity of tubercle bacilli. Am Rev Respir Dis 1967; 95: 461-469.

5. McDermott W, Tomsett R. Activation of pyrazinamide and nicotinamide in acid environments in vitro. Am Rev Tuberc 1954; 70: 748-754.

6. Heifets L, Lindholm-Levy P. Pyrazinamide sterilizing activity in vitro against semi-dormant Mycobacterium tuberculosis bacterial populations. Am Rev Respir Dis 1992; 145: 12231225.

7. Salfinger M, Heifets L. Determination of pyrazinamide MICs for Mycobacterium tuberculosis at different $\mathrm{pHs}$ by the radiometric method. Antimicrob Agents Chemother 1988; 32: $1002-1004$.

8. Stottmeier KD, Beam RE, Kubica GP. Determination of drug susceptibility of mycobacteria to pyrazinamide in $7 \mathrm{H} 10$ agar. Am Rev Respir Dis 1967; 96: 1072-1075.

9. Siddiqi S. Radiometric (BACTEC) tests for slowly growing mycobacteria. In: Isenberg $\mathrm{H}$ (ed) Clinical microbiology procedures handbook. Washington, DC, American Society for Microbiology. 1992: 1-25.

10. Butler WR, Kilburn JO. Improved method for testing susceptibility of Mycobacterium tuberculosis to pyrazinamide. J Clin Microbiol 1982; 16: 1106-1109.

11. Butler WR, Kilburn JO. Susceptibility of Mycobacterium tuberculosis to pyrazinamide and its relationship to pyrazinamidase activity. Antimicrob Agents Chemother 1983; 24: 600601.

12. Heifets LB. Drug susceptibility tests in the management of chemotherapy of tuberculosis. In: Heifets LB, Cynamon $\mathrm{MH}$ (eds) Drug susceptibility in the chemotherapy of mycobacterial infections. Boca Raton, CRC Press. 1991: 89-122.

13. Heifets LB. Drug susceptibility testing in mycobacteriology. In: Heifets LB (ed) Clinical mycobacteriology Philadelphia, WB Saunders. 1996: 641-656.

14. Heifets L, Sanchez T. New agar medium for testing susceptibility of Mycobacterium tuberculosis to pyrazinamide. $J$ Clin Microbiol 2000; 38: 1498-1501.

15 Centers for Disease Control. Nosocomial transmission of multidrug-resistant tuberculosis among HIV-infected persons Florida and New York, 1988-1991. MMWR Morb Mortal Wkly Rep 1991; 40: 585-591.

16. Hewlett D, Horn DL, Alfalla C. Drug-resistant tuberculosis: inconsistent results of pyrazinamide susceptibility testing. JAMA 1995; 273: 916-917.

17. Miller MA, Thibert L, Desjardins F, Siddiqi SH, Dascal A. Growth inhibition of Mycobacterium tuberculosis by polyoxyethilene stearite present in the BACTEC pyrazinamide susceptibility test. J Clin Microbiol 1996; 34: 84-86.

18. Zhang Y, Permar S, Sun Z. Conditions that may affect the results of Mycobacterium tuberculosis susceptibility testing to pyrazinamide. J Med Microbiol 2002; 50: 43-50.

19. Cheng S-J, Thibert L, Sanchez T, Heifets L, Zhang Y. pncA mutations as a major mechanism of pyrazinamide resistance in Mycobacterium tuberculosis: spread of a monoresistant strain in Quebec, Canada. Antimicrob Agents Chemother 2000; 44: $528-532$. 